\title{
Long-Term Effects of Pedometer-Based Physical Activity Coaching in Severe COPD: A Randomized Controlled Trial
}

This article was published in the following Dove Press journal: International Journal of Chronic Obstructive Pulmonary Disease

\author{
Dario Kohlbrenner (D) \\ Noriane A Sievi $\mathbb{B D}^{\prime}$ \\ Oliver Senn (D) $^{2}$ \\ Malcolm Kohler' \\ Christian F Clarenbach (1D) \\ 'Department of Pulmonology, University \\ Hospital Zurich, Zurich, Switzerland; \\ ${ }^{2}$ Institute of Primary Care, University of \\ Zurich, Zurich, Switzerland
}

Background and Objective: Limited evidence on long-term effects of physical activity programs in COPD is available. The aim of the study was to investigate the effects of a threemonth program combining physical activity counselling and pedometer-based feedback in addition to usual care, followed by a nine-month unsupervised observation period as compared to usual care in participants with severe to very severe COPD.

Methods: Participants were randomized to either a control group receiving usual care or an intervention group receiving motivational support, an activity diary with an individual step count goal (ie, an increase of $\geq 15 \%$ from baseline) and a pedometer in addition to usual care. The intervention ended after three months and an unsupervised observational period followed until twelve months. Primary outcome was daily step count after one year.

Results: Seventy-four participants were included, 61 (82\%) completed the study. Linear regression modelling, adjusted for baseline step count, showed no significant difference in change in step count after 12 months between the groups $(\mathrm{B}=547.33,95 \% \mathrm{CI}=-243.55 / 1338.20)$.

Conclusion: A three-month program combining physical activity counselling and pedometer-based feedback in addition to usual care does not attenuate the declining course of physical activity in participants with severe and very severe COPD during a long term follow-up of one year as compared to usual care. This result was primarily determined by the low intervention response rates to the combined program.

Clinical Trial Registration: www.ClinicalTrials.gov, NCT03114241.

Keywords: physical activity, COPD, counselling program, randomized controlled trial, long-term follow-up

\section{Plain Language Summary}

Combined physical activity counselling and pedometer-based feedback is a promising intervention towards physical activity enhancement in participants with COPD. However, scarce long-term data on its effects are available. In this study, we investigated the effects of three months combined physical activity counselling and pedometer-based feedback, followed by a nine-month unsupervised observation period as compared to usual care in participants with severe to very severe COPD. We found that the three-month combined program was not superior to usual care in preserving physical activity after one year. Interestingly, a similar number of participants in both groups managed to improve their physical activity.

\section{Introduction}

Physical activity (PA) is an important predictor and prognostic factor of health status in apparently healthy populations. ${ }^{1}$ It is well recognized that changes in PA
Correspondence: Christian F Clarenbach Department of Pulmonology, University Hospital Zurich, Raemistrasse 100, Zurich 8091, Switzerland

Tel +4I 44255 I7 I2

Email christian.clarenbach@usz.ch
International Journal of Chronic Obstructive Pulmonary Disease 2020:I5 2837-2846

2837

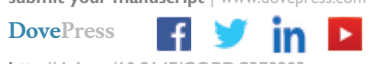

http://doi,org/10.2147/CO 
behavior ameliorate health outcomes. ${ }^{1}$ In chronic obstructive pulmonary disease (COPD), low PA levels are common and a known risk factor for worsening disease status (ie, increased risk of COPD-related hospitalization and mortality), ${ }^{2}$ while peripheral muscle dysfunction progressively exacerbates the deficiency. ${ }^{3}$ Despite this, access to and uptake of multimodal rehabilitation is $\operatorname{low}^{4-6}$ and may only partially recover PA impairment. ${ }^{2}$

Considering the broad applicability and relatively low cost of PA programs makes them appealing for implementation in the COPD population. Accordingly, programs targeting PA directly are a promising treatment option. However, a recent Cochrane review found conflicting evidence regarding the effectiveness of various interventions aiming to increase PA in COPD. ${ }^{7}$ The included studies used a wide range of different interventions, devices and outcomes, hampering comparison. ${ }^{7}$ Among the various interventions investigated, combining PA counselling with pedometer-based feedback showed the most promising results. ${ }^{8}$ There is consensus that the measurement of PA needs continuous and accurate monitoring to provide valuable feedback, which state-of-the-art pedometers and accelerometers can achieve nowadays. In addition, they have become widely available and affordable, enabling broad application in clinical practice. Looking at the available evidence on combined PA counselling and pedometer-based feedback in detail, participants with a baseline PA of less than $10^{\prime} 000$ steps per day seem to profit most. ${ }^{9}$ Furthermore, limited evidence on the longterm effects of such programs is available. ${ }^{2,10}$ Building on these findings, the combined program may be suitable for participants with more severe COPD, commonly experiencing more pronounced PA impairment and more severely impaired participation in activities of daily living.

Thus, we aimed to investigate the effects of a threemonth program combining PA counselling and pedometerbased feedback in addition to usual care, followed by a nine-month unsupervised observation period as compared to usual care in participants with severe to very severe COPD. Furthermore, we aimed to explore predictors enabling these participants to accomplish an increase in PA.

\section{Materials and Methods}

\section{Study Subjects}

Data collection ran between May 2017 and May 2020. Participants aged 40 years or older with confirmed severe and very severe COPD (ie, $\mathrm{FEV}_{1}<50 \%$ pred.) according to the Global Initiative for Chronic Obstructive Lung Disease (GOLD)-guidelines ${ }^{11}$ were assessed for eligibility out of outpatient pulmonary clinic patient pools and approached during outpatient visits or via letter. Participants with diagnosed mental or physical disability precluding informed consent or compliance with the protocol, experiencing acute or recent (ie, within the last 6 weeks) exacerbation of COPD, attending pulmonary rehabilitation within the last 6 months and pregnant participants were not included.

\section{Study Design}

A single-center parallel group, randomized controlled trial was performed at the Department of Pulmonology, University Hospital Zurich, Switzerland. Randomization was performed on a 1:1 ratio using sealed envelopes.

The study was conducted in accordance with the declaration of Helsinki and all subjects provided written informed consent. The Ethics Committee of the Canton of Zurich approved the study (EK-ZH-NR: 2016-00151), and the study is registered on www.ClinicalTrials.gov, NCT03114241.

\section{Methods}

\section{Experimental Design}

Participants were randomized to either a control group receiving usual care or an intervention group receiving PA counselling and pedometer-based feedback in addition to usual care (ie, regular visits at their respiratory physician).

Participants allocated to the intervention group received a pedometer (HJ-325-EB, Omron Healthcare Co., Kyoto, Japan) and used it as a motivational and feedback tool. Furthermore, they were provided an activity diary concerning their daily activities, step counts and factors influencing their PA. PA counselling consisted of a familiarization session and individually determined daily PA goal setting (ie, an increase in step count of $15 \%$ compared to the baseline value). ${ }^{12}$ Thereafter, monthly telephone calls were performed until the three-month follow-up visit. The calls were of motivational nature; addressing the diary of the previous month, encouraging protocol compliance and supporting participants in identifying and coping with barriers towards enhanced PA. Sessions and phone calls were performed by the same investigator, a respiratory physiotherapist, ensuring consistency. The PA counselling program ended after three 
months. Following the study visit, participants kept their pedometer and were encouraged to sustain their increased level of PA until the end of the study at 12 months. No further motivational calls or support were provided.

\section{Daily Physical Activity}

The number of steps per day, an indicator for PA, was measured through a validated, triaxial accelerometer of a multisensory activity monitor (SenseWear Pro ${ }^{\text {TM}}$; Bodymedia Inc., Pittsburgh, PA, USA). ${ }^{13}$ The monitor was worn on the upper left arm for seven consecutive days at baseline, at three and at 12 months (end of study) by both groups. Participants were instructed to wear the device for 24 hours a day, except while showering or swimming. The threshold for valid data from the armband was set at a usage time of 4 days with a minimum of 22.5 hours per day. ${ }^{2}$ Seasonality was assessed by the season during which the visit took place (ie, summer, autumn, winter, and spring).

\section{Respiratory Variables}

All participants underwent pulmonary function testing according to the American Thoracic Society and European Respiratory Society (ATS/ERS) guidelines to measure forced expiratory volume in one second $\left(\mathrm{FEV}_{1}\right)$, forced vital capacity (FVC), and lung diffusion capacity of carbon monoxide (TLCO). ${ }^{14,15}$ Values were obtained after shortacting bronchodilator application. The tests were performed by pulmonary function laboratory staff being unaware of the study protocol and blinded to group allocation.

\section{Exacerbations}

Acute exacerbations of COPD (AECOPD) were defined as increases in dyspnea, cough and/or sputum production leading to the prescription of antibiotics and/or corticosteroids. AECOPD requiring hospital admission were defined as severe acute exacerbations of COPD (SAECOPD). Data on AECOPD and SAECOPD during the study was acquired through the comparison of participant reports and medical records from the general practitioner, pulmonologist and hospital.

\section{COPD-Specific Health Status}

Subjective health status capturing COPD symptom burden was recorded by the COPD assessment test (CAT), a valid and reliable questionnaire for the COPD population. ${ }^{16}$

\section{Functional Exercise Capacity}

The 1-minute sit-to-stand test (1MSTS) was performed using a standardized protocol ${ }^{17-19}$ on a conventional chair without armrest and a seat height of $46 \mathrm{~cm}$. For safety reasons, the chair was positioned against a wall. Participants were instructed to stand-up and sit-down as often as possible at a self-chosen speed during one minute and the number of sit-to-stand repetitions was counted. Participants were told to place their hands at the hips and were not allowed to use them to assist movement. Verbal encouragement was not provided during the test, except the information when $15 \mathrm{~s}$ were left until the test terminated after $60 \mathrm{~s} .{ }^{17-20}$

\section{Analysis}

All results are shown as mean (SD) unless otherwise stated, normal distribution of the variables was determined visually using quantile-quantile plots. A two-sided p-value of $<0.05$ was considered to be statistically significant. Data were analyzed according to the intention-to-treat principle.

The primary outcome of the study was change in daily step count, an indicator for PA, from baseline to 12 months after inclusion. Sample size calculation was planned to detect a difference in daily step count of 725 (1000) steps per day between the groups at study end and is based on data from our previous cohort. ${ }^{21}$ Calculation determined 37 participants per group to be sufficient, yielding at a power of $80 \%$, setting $\alpha$ to 0.05 and accounting for a dropout rate of $15 \%$.

For the analysis of the primary outcome, a linear regression model containing the change in daily step count between baseline and 12-month follow-up as the dependent variable and group allocation as an independent variable was fitted. The model was adjusted for differences in baseline step count between the study groups. The same model was applied to the sample when stratified according to an increase in PA by $\geq 15 \%$ at 3 months.

Predictors towards an increase in PA by $\geq 15 \%$ were analyzed using a linear regression model containing the change in daily step count between baseline and 3-month follow-up as the dependent variable and group allocation, age, sex, body-mass-index, smoking status, pack years, $\mathrm{FEV}_{1}$, CAT-score, seasonality, and number of exacerbations as independent variables.

Statistical analysis was performed using $\mathrm{R}$ ( $\mathrm{R}$ Core Team 2019, R Foundation for Statistical Computing, Vienna, Austria).

\section{Results}

Seventy-four participants were included, of whom 61 $(82 \%)$ completed the study, see Figure 1. The sample 
had a mean age of 66 (9) years, was mainly male $(68 \%)$ and diagnosed with severe COPD $\left(\mathrm{FEV}_{1} \%\right.$ pred. 35.04 [9.92] \%). There were no apparent differences in baseline characteristics between the groups regarding age, sex, body-mass index, smoking status, pack years and airflow obstruction, for complete baseline patient characteristics see Table 1. The intervention group showed clinically relevant higher mean daily step counts and repetitions in the 1MSTS at baseline compared to the control group.

Participants discontinuing the study did not differ from study completers in terms of baseline characteristics listed in Table 1.

Mean step count at baseline in the intervention group was 3708 (3601) steps, and 2451 (1819) in the control group, respectively. After three months 4462 (4955) steps in the intervention, and 2883 (2547) in the control group, respectively. At the primary endpoint after 12 months, step count was 4101 (3997) steps in the intervention, and 2137 (2547) in the control group, respectively (see Figure 2). The absolute change in step count after 12 months was -108 (1057) steps in the intervention, and -480 (1703) steps in the control group, respectively. The changes in step count according to group allocation are shown in Figure 3. Linear regression modelling, adjusted for baseline step count, showed no significant difference in change in step count after 12 months between the groups $(B=547.33,95 \% \mathrm{CI}=$ $-243.55 / 1338.20, \mathrm{p}=0.171)$. Results of linear regression modelling are displayed in Table 2.

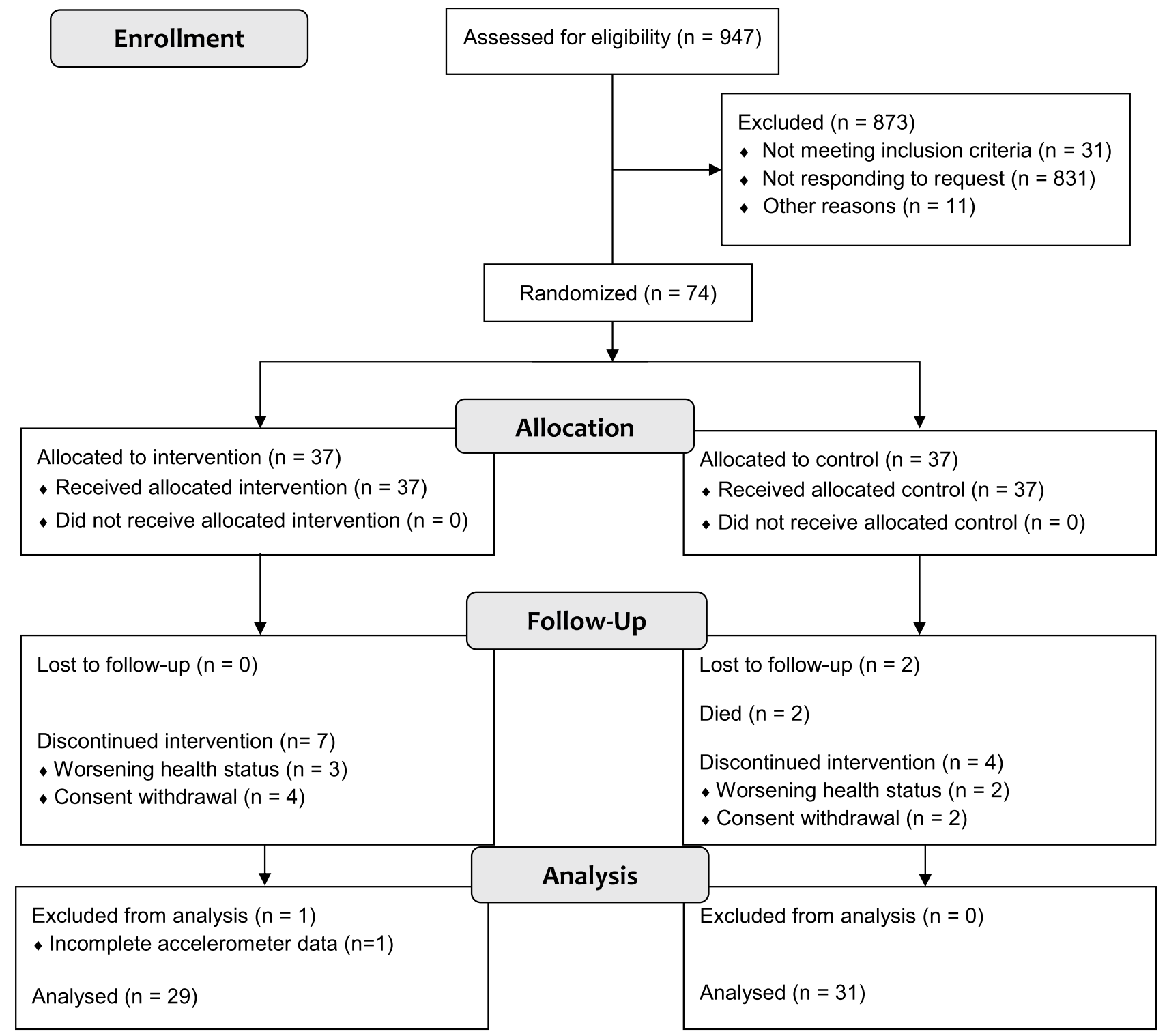

Figure I Study participant flow diagram. 
Table I Patient Characteristics Stratified per Group

\begin{tabular}{|l|l|l|}
\hline & Control & Intervention \\
\hline $\mathrm{n}$ & 37 & 37 \\
Age, y & $67.05(8.5 \mathrm{I})$ & $64.35(9.36)$ \\
BMI, kg/m ${ }^{2}$ & $27 / 10(73 / 27)$ & $23 / 14(62 / 38)$ \\
Smoking status, yes/no (\%) & $7 / 30(19 / 8 \mathrm{I})$ & $5 / 32(14 / 86)$ \\
Pack Years, N & $59(29.26)$ & $46(27.34)$ \\
\hline COPD Risk Group, N (\%) & & \\
A & $1(2.7)$ & $4(10.8)$ \\
B & $24(64.9)$ & $19(51.4)$ \\
C & $0(0)$ & $0(0)$ \\
D & $12(32.4)$ & $14(37.8)$ \\
\hline CAT Score, N & $19.14(6.15)$ & $17.14(6.77)$ \\
FEV , I & $1.07(0.31)$ & $0.98(0.42)$ \\
FEV, \% pred. & $35.89(8.82)$ & $34.19(10.97)$ \\
FVC, I & $2.63(0.56)$ & $2.48(0.82)$ \\
FVC, \% pred. & $68.19(13.22)$ & $67.59(15.29)$ \\
Daily step count, N & $2450.74(1819.12)$ & $3707.80(3601.26)$ \\
IMSTS repetitions, N & $16.06(8.72)$ & $20.97(7.04)$ \\
\hline
\end{tabular}

Note: Data are mean (SD) or $\mathrm{n}(\%)$ unless otherwise stated.

Abbreviations: BMI, body mass index; CAT, COPD assessment test; $\mathrm{FEV}_{1}$, forced expiratory volume in one second; pred., predicted; FVC, forced vital capacity; IMSTS, I-minute sit-to-stand test.

Stratifying the participants according to their change in PA of $\geq 15 \%$ or $<15 \%$ until the three-month follow-up showed that equal amounts of participants per group increased their baseline step count $\geq 15 \%$ (13 in the intervention vs 14 in the control group). A majority of participants in both groups did not increase their step count above the threshold ( 20 in the intervention vs 19 in the control group).

A statistically significant and clinically relevant difference in change in step count after 12 months, adjusted for baseline step count, was observed when stratifying the sample according to an increase $\geq 15 \%$ in PA at 3 months $(\mathrm{B}=1105.58,95 \% \mathrm{CI}=376.87 / 1834.30, \mathrm{p}=0.004)$. The absolute change in step count after 12 months was 303 (1170) steps in the ones increasing PA $\geq 15 \%$, and -742 (1472) steps in the ones not doing so, respectively. The course of step count according to PA change is shown in Figure 4. The linear regression model identified younger age as a significant predictor $(B=-111.92,95 \% \quad C I=-212.34 /-$ $11.50, \mathrm{p}=0.03$ ) towards a positive response (ie, increase in daily step count $\geq 15 \%$ from baseline) in the control group. Regarding the responders in the intervention group, the model identified a higher baseline step count $(\mathrm{B}=0.31,95 \%$ $\mathrm{CI}=0.13 / 0.48, \mathrm{p}=0.001$ ) as a significant predictor. Results of linear regression modelling assessing predictor variables is shown in Table 3. Severity of airflow obstruction overall declined by -0.09 (0.17) 1 during one year.

Regarding secondary outcomes, CAT-score differences from baseline to 12 months were not clinically relevant with -1.6 (8.3) in the intervention, and -1.3 (7.5) points in the control group, respectively. The 1MSTS repetition differences were -0.5 (6.9) in the intervention, and 1.0 (7.2) repetitions in the control group, respectively. Absolute differences in primary and secondary outcomes are displayed in Table 4.

\section{Discussion}

Our study found no relevant difference regarding the change in step count after one year between a group receiving a three-month program combining PA counselling and pedometer-based feedback in addition to usual care, followed by a nine-month unsupervised period in comparison to a control group receiving usual care. A comparable number of participants in both groups increased their step count by $\geq 15 \%$ up to three months after baseline.

Our findings do not support the application of the described intervention in participants with severe and very severe COPD. The design consisted of limited counselling contacts and easy-to-handle pedometers aiming to provide a widely accessible and implementable program at scalable cost. Similar approaches were previously applied in two studies including participants with mild to moderate COPD. ${ }^{8,9}$ Of which one found promising short-term results. ${ }^{8}$ The second found matching long-term results to ours in the subgroup recruited from primary care. ${ }^{9}$ Regular phone calls and activity diaries have previously been shown to increase short-term PA in severe COPD. ${ }^{22}$ However, they used more frequent phone calls during a very short intervention period (ie, two weeks). ${ }^{22}$ It remains to be studied if a similar intervention to ours with more frequent counselling via phone would alter the results. However, interventions providing feedback, motivational support and diaries in an internet-based manner found matching results to our investigation. ${ }^{23,24}$ The more frequent and easily accessible feedback accordingly does not seem to enhance response to the physical activity intervention.

Interestingly, a comparable number of participants in both groups increased their step count to or above the cutoff. The consequent stratified analysis showed statistically and clinically relevant lower changes in daily step count after 12 months in participants having increased their PA 


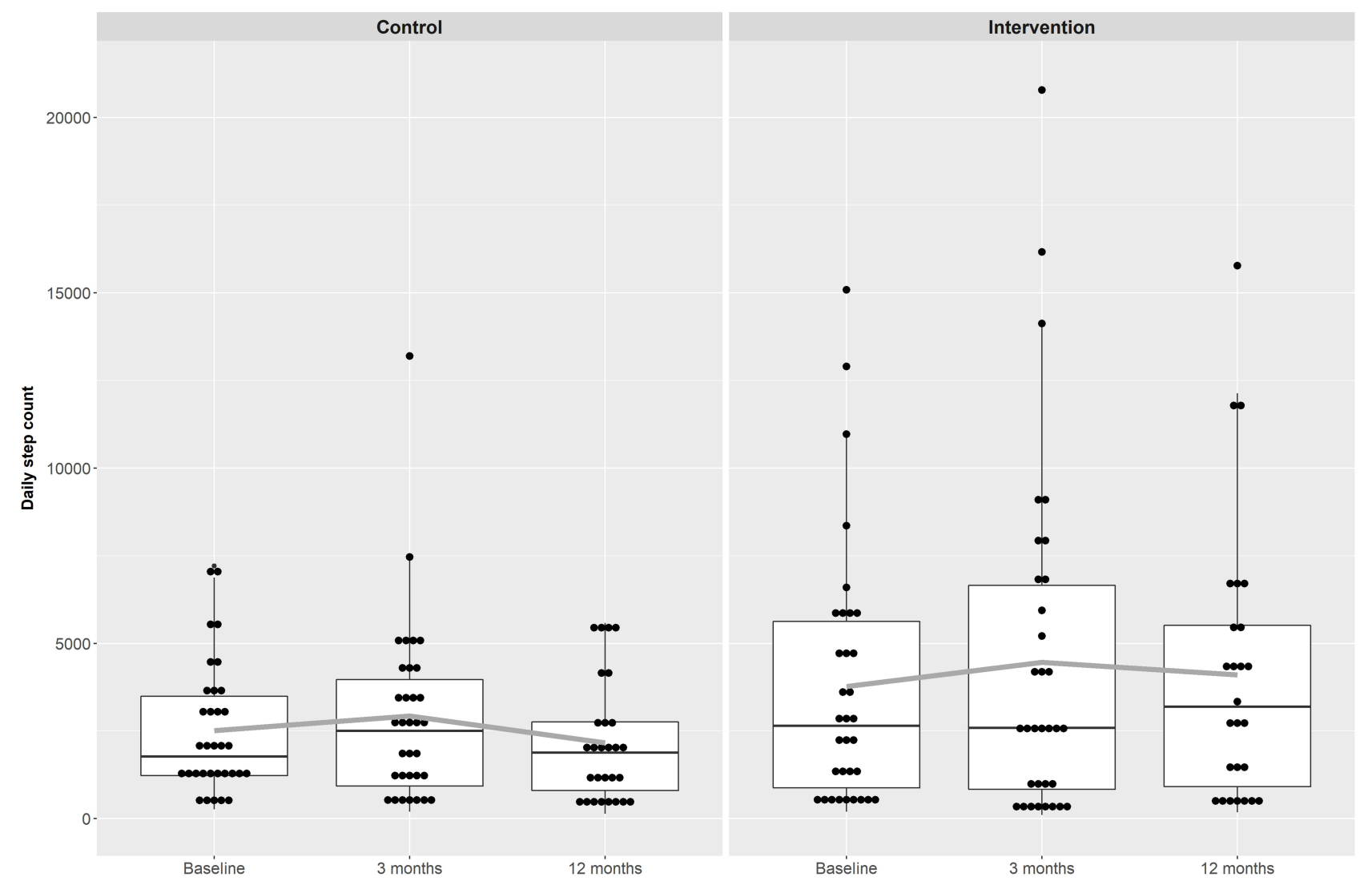

Figure 2 Boxplots showing daily step count at baseline and 3-and 12-month follow-up visits in control group (difference from baseline to end of study - 108 (1057) steps) and intervention group ( -480 (1703) steps), $p=0.342$. Data are mean (SD). Solid grey line: mean.

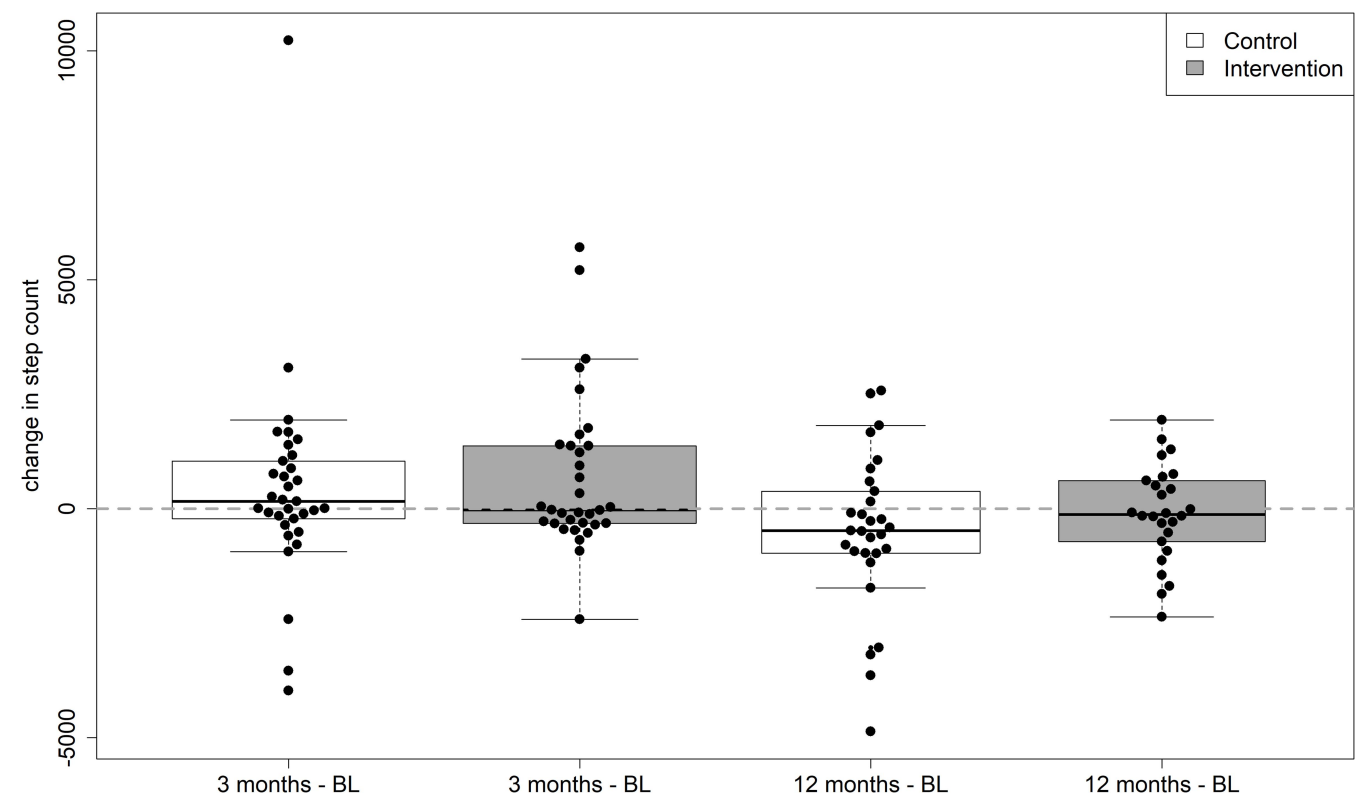

Figure 3 Boxplots showing the changes in daily step count compared to baseline values at 3- and I2-month follow-ups stratified according to group allocation. Abbreviation: BL, baseline. 
Table 2 Linear Regression Model Results for Step Count After 12 Months

\begin{tabular}{|l|l|}
\hline Dependent Variable & Coefficient \\
\hline Group allocation & $547.33(-243.55 / / 338.20,0.17 I)$ \\
Increase in PA $\geq 15 \%$ & $I 105.58(376.87 / / 834.30,0.004)$ \\
\hline
\end{tabular}

Notes: Data are B ( $95 \%$ confidence interval, p-value). Group allocation: stratified according to intention-to-treat; increase in PA $\geq 15 \%$ : stratified according to increase in $\mathrm{PA} \geq 15 \%$ or below.

Abbreviation: PA, physical activity.

$\geq 15 \%$ at three months, suggesting that this threshold is effective in terms of preserving PA over one year. Comparing the observed preservation of PA to an observational study, a benefit of $>1600$ steps per day upon response is assumed. ${ }^{21}$ On the one hand, our findings suggest a low response rate or adherence to the intervention under investigation. On the other hand, they indicate that a certain phenotype of participants might be responsive. More specifically, 14 participants in the control group responded to the usual care setting (ie, information from the treating physician that increased PA is beneficial) with an increase in PA to or above the defined threshold. Aiming to identify predictors towards this beneficial behavior, our linear regression model suggested that younger age and higher baseline PA enable a response towards increased PA.

Participants with severe and very severe COPD experience impairment in multiple dimensions, such as PA, exercise capacity, and health-related quality-of-life. Our findings are in line with previous investigations, ${ }^{10}$ suggesting that a one-dimensional target might not be sufficient to achieve relevant and sustained PA changes in this population. However, in some very specific patient groups simple PA counselling may be sufficient, while others do not respond to a combined intervention (ie, PA counselling and pedometer-based feedback). We suggest to adapt PA interventions more closely to various phenotypes which have to be investigated in further studies. Considering the high behavioral contribution towards a change in PA, ${ }^{10}$ a multidimensional and possibly repeated intervention might be required in severely diseased participants. Additionally, a certain threshold of exercise tolerance

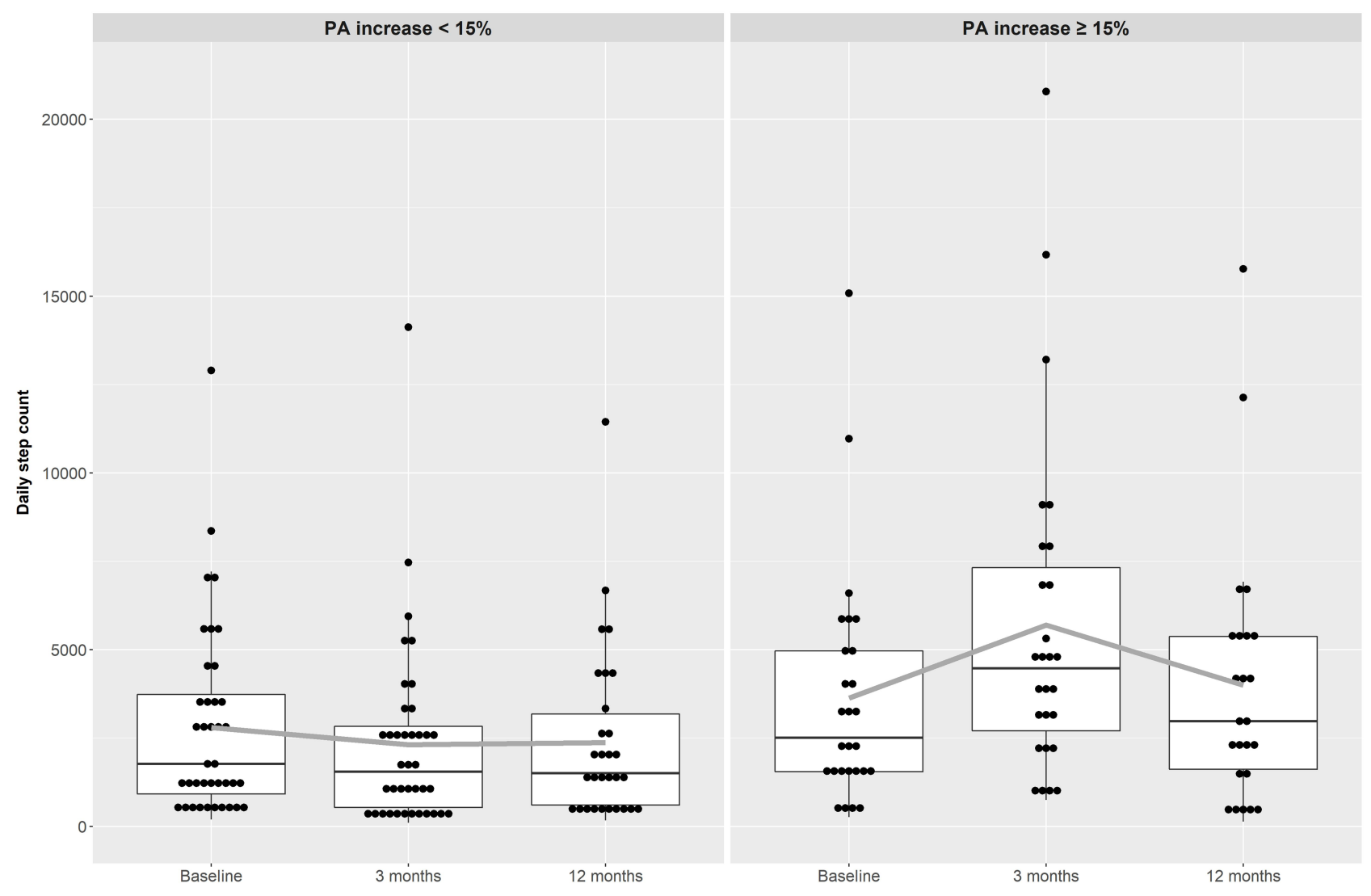

Figure 4 Boxplots showing daily step count at baseline and 3- and 12-month follow-up visits in participants increasing their PA $\geq 15 \%$ (difference from baseline to end of study 303 (1 I70) steps) and participants increasing their PA < 15\% or decreasing their PA ( 742 (I472) steps), P = 0.007. Data are mean (SD). Solid grey line: mean. Abbreviation: PA, physical activity. 
Table 3 Linear Regression Model Assessing Predictors Towards Increased PA at 3 Months Stratified According to Group Allocation

\begin{tabular}{|c|c|}
\hline Predictor Variables & Coefficient \\
\hline \multicolumn{2}{|l|}{ Control } \\
\hline $\begin{array}{l}\text { Age } \\
\text { Sex, male } \\
\text { BMI } \\
\text { Smoking status, yes } \\
\text { Pack years } \\
\text { FEV }_{1}, \text { I } \\
\text { Exacerbations, } n \\
\text { Baseline step count }\end{array}$ & $\begin{array}{l}-|I| .9 \mid(-2|2.34 /-| \mid .50,0.030) \\
-599.80(-2456.57 /|256.96,0.5| I) \\
\text { I34.50(-83.6I/352.60, 0.2I5) } \\
400.08(-|809.76 / 2609.9|, 0.7 \mid I) \\
-15.40(-38.65 / 7.84,0.184) \\
756.18(-2068.08 / 3580.45,0.586) \\
69.48(-657.76 / 796.7 \mid, 0.845) \\
-0.29(-0.7 \mid / 0.13,0.162)\end{array}$ \\
\hline \multicolumn{2}{|l|}{ Intervention } \\
\hline $\begin{array}{l}\text { Age } \\
\text { Sex, male } \\
\text { BMI } \\
\text { Smoking status, yes } \\
\text { Pack years } \\
\text { FEV , I } \\
\text { Exacerbations, } n \\
\text { Baseline step count }\end{array}$ & $\begin{array}{l}10.70(-52.80 / 74.19,0.731) \\
131.74(-1 \mid 121.64 / 1385.12,0.830) \\
28.68(-9 \mid .88 / 149.23,0.628) \\
386.54(-1319.56 / 2092.64,0.644) \\
1.34(-19.97 / 22.64,0.900) \\
35.51(-\mid 779.25 / 1850.26,0.970) \\
-14.67(-247.52 / 2 \mid 8.18,0.900) \\
0.31(0.13 / 0.48,0.001)\end{array}$ \\
\hline
\end{tabular}

Note: Data are B (95\% confidence interval, p-value).

Abbreviations: BMI, body mass index; $\mathrm{FEV}_{1}$, forced expiratory volume in one second; I, liters.

Table 4 Differences from Baseline to 3-Month and 12-Month Follow-Up Between Groups

\begin{tabular}{|l|l|l|l|}
\hline & Control & Intervention & p-value \\
\hline CAT score 3 months & $-1.92(4.45)$ & $-0.86(5.18)$ & 0.357 \\
CAT score 12 months & $-1.63(8.28)$ & $-1.32(7.49)$ & 0.876 \\
Daily step count 3 months & $423(2258)$ & $694(1709)$ & 0.585 \\
Daily step count I2 months & $-480(1703)$ & $-108(1057)$ & 0.342 \\
IMSTS repetitions 3 months & $1.81(5.97)$ & $0.74(3.46)$ & 0.418 \\
IMSTS repetitions I2 months & $-0.5(6.9)$ & $1.0(7)$ & 0.478 \\
\hline
\end{tabular}

Note: Data are mean (SD).

Abbreviations: CAT, COPD assessment test; IMSTS, I-minute sit-to-stand test.

seems necessary to improve PA. ${ }^{10,25}$ Since pulmonary rehabilitation is effective in terms of increasing exercise tolerance, ${ }^{26}$ further studies may investigate PA counselling programs including pedometer-based feedback implemented or subsequent to such.

This study has some limitations, while pedometers are known to accurately measure step counts, precision attenuates in slow walkers. ${ }^{27}$ Participants with severe COPD may be severely impaired in walking and our PA measure might therefore underestimate their step count. However, we used a validated device in the COPD population for our primary outcome.
Furthermore, self-efficacy or motivation towards a behavioral change might be a predictor towards treatment response, which we did not systematically assess. However, engaging in a PA study already forms a motivational statement. Complementing this, enrolment was low with 831 participants not responding to a participation request. We may only speculate about the reasons therefore. Our inclusion criteria with a rather strict attempt to control for effects resulting from exacerbation and pulmonary rehabilitation might restrict eligible participants. In addition, the long follow-up period might reduce excitement for participation. The dropout rate of 13 participants surpassed the assumed rate of $15 \%$ by $3 \%$. However, participants who discontinued the study were not different in terms of baseline characteristics as compared to the ones finishing the study.

\section{Conclusions}

In conclusion, a three-month program combining PA counselling and pedometer-based feedback in addition to usual care has no impact on the course of PA in participants with severe and very severe COPD during a long term followup of one year as compared to usual care. This result was primarily determined by the low intervention response rates to the combined program. Our results suggest that low-frequency PA counselling in combination with pedometer-based feedback should not be implemented into clinical practice of participants with severe to very severe COPD. Future research might integrate more recent advances (such as web-platforms, home-monitoring) in targeting PA impairment in COPD.

\section{Abbreviations}

PA, physical activity; COPD, chronic obstructive pulmonary disease; GOLD, Global Initiative for Chronic Obstructive Lung Disease; ATS/ERS, European Respiratory Society/ American Thoracic Society; $\mathrm{FEV}_{1}$, forced expiratory volume in one second; FVC, forced vital capacity; TLCO, lung diffusion capacity of carbon monoxide; AECOPD, acute exacerbation of COPD; SAECOPD, severe acute exacerbations of COPD; CAT, COPD assessment test; 1MSTS, 1-minute sit-to-stand test.

\section{Data Sharing Statement}

The data supporting the findings of this study are available upon reasonable request to the corresponding author. 


\section{Acknowledgments}

We thank Marc Spielmanns, MD and Sabine Schöndorf from the Department of Pulmonary Rehabilitation, RehaZentren Zürich, Klinik Wald; Jean-Luc Kurzen, MD from the Department of Pulmonology, Spital Männedorf; Christian Alfaré, MD from the Department of Pulmonology, Spital Uster; Eva Achermann, MD from the Department of Pulmonology, Spital Limmattal; and Stephan Wieser, MD from the Department of Pulmonology, Stadtspital Waid for their support during the recruitment stage. We thank Sandra Siegfried, MSc from the Epidemiology, Biostatistics and Prevention Institute at the University of Zurich for statistical assistance during the analysis stage.

\section{Author Contributions}

All authors contributed to data analysis, drafting or revising the article, have agreed on the journal to which the article will be submitted, gave final approval of the version to be published, and agree to be accountable for all aspects of the work.

\section{Funding}

This work was supported by Lunge Zurich.

\section{Disclosure}

M. Kohler reports personal fees from Bayer, Astra Zeneca, Boehringer Ingelheim, Novartis, Roche, CSL Behring, and Mundipharma, outside the submitted work. M. Kohler is member of the board of the Deep Breath Initiative (DBI). A company that provides services in the field of breath analysis C.F. Clarenbach reports personal fees from Roche, Novartis, Boehringer Ingelheim, GSK, Astra Zeneca, Sanof, Vifor, and Mundipharma, outside the submitted work.

The authors report no other potential conflicts of interest for this work.

\section{References}

1. World Health Organization. Global Recommendations on Physical Activity for Health. Geneva: World Health Organization; 2010.

2. Watz H, Pitta F, Rochester CL, et al. An official European Respiratory Society statement on physical activity in COPD. Eur Respir J. 2014;44 (6):1521-1537. doi:10.1183/09031936.00046814

3. Maltais F, Decramer M, Casaburi R, et al. An official American Thoracic Society/European Respiratory Society statement: update on limb muscle dysfunction in chronic obstructive pulmonary disease. $\mathrm{Am}$ J Respir Crit Care Med. 2014;189(9):e15-62.

4. Puhan M, Koller M, Brandli O, Steurer J. [Pulmonary rehabilitation of COPD in Switzerland-an assessment of current status]. Praxis. 2003;92(4):111-116.doi:10.1024/0369-8394.92.4.111. German.
5. Rochester CL, Holland AE, Rehabilitation P. Improved survival for patients with COPD. JAMA. 2020;323(18):1783-1785. doi:10.1001/ jama.2020.4436

6. Spruit MA, Van't Hul A, Vreeken HL, et al. Profiling of patients with COPD for adequate referral to exercise-based care: the Dutch model. Sports Med. 2020;50(8):1421-1429. doi:10.1007/s40279-020-01286-9

7. Burge AT, Cox NS, Abramson MJ, Holland AE. Interventions for promoting physical activity in people with chronic obstructive pulmonary disease (COPD). Cochrane Database Syst Rev. 2020;4(4): Cd012626.

8. Mendoza L, Horta P, Espinoza J, et al. Pedometers to enhance physical activity in COPD: a randomised controlled trial. Eur Respir J. 2015;45(2):347. doi:10.1183/09031936.00084514

9. Altenburg WA, Ten Hacken NH, Bossenbroek L, Kerstjens HA, de Greef MH, Wempe JB. Short- and long-term effects of a physical activity counselling programme in COPD: a randomized controlled trial. Respir Med. 2015;109(1):112-121. doi:10.1016/j.rmed.2014.10.020

10. Troosters T, Blondeel A, Rodrigues FM, Janssens W, Demeyer H. Strategies to increase physical activity in chronic respiratory diseases. Clin Chest Med. 2019;40(2):397-404. doi:10.1016/j.ccm.2019.02.017

11. Global Initiative for Chronic Obstructive Lung Disease. Global strategy for the diagnosis, management, and prevention of chronic obstructive pulmonary disease (2018 report); 2018.

12. Nolan CM, Maddocks M, Canavan JL, et al. Pedometer step count targets during pulmonary rehabilitation in chronic obstructive pulmonary disease. A randomized controlled trial. Am J Respir Crit Care Med. 2017;195(10):1344-1352. doi:10.1164/rccm.201607-1372OC

13. Van Remoortel H, Raste Y, Louvaris Z, et al. Validity of six activity monitors in chronic obstructive pulmonary disease: a comparison with indirect calorimetry. PLoS One. 2012;7(6):e39198. doi:10.1371/journal.pone. 0039198

14. Miller MR, Hankinson J, Brusasco V, et al. Standardisation of spirometry. Eur Respir J. 2005;26(2):319-338. doi:10.1183/ 09031936.05.00034805

15. Macintyre N, Crapo RO, Viegi G, et al. Standardisation of the single-breath determination of carbon monoxide uptake in the lung. Eur Respir J. 2005;26(4):720-735. doi:10.1183/09031936.05.00034905

16. Jones PW, Harding G, Berry P, Wiklund I, Chen WH, Kline Leidy N. Development and first validation of the COPD assessment test. Eur Respir J. 2009;34(3):648-654. doi:10.1183/09031936.00102509

17. Crook S, Busching G, Schultz K, et al. A multicentre validation of the 1-min sit-to-stand test in patients with COPD. Eur Respir J. 2017;49(3):1601871. doi:10.1183/13993003.01871-2016

18. Radtke T, Hebestreit H, Puhan MA, Kriemler S. The 1-min sit-tostand test in cystic fibrosis - Insights into cardiorespiratory responses. J Cyst Fibros. 2017;16(6):744-751. doi:10.1016/j.jcf.2017.01.012

19. Strassmann A, Steurer-Stey C, Lana KD, et al. Population-based reference values for the 1-min sit-to-stand test. Int J Public Health. 2013;58(6):949-953. doi:10.1007/s00038-013-0504-z

20. Radtke T, Puhan MA, Hebestreit H, Kriemler S. The 1-min sit-tostand test-a simple functional capacity test in cystic fibrosis? J Cyst Fibros. 2016;15(2):223-226. doi:10.1016/j.jcf.2015.08.006

21. Clarenbach CF, Sievi NA, Haile SR, et al. Determinants of annual change in physical activity in COPD. Respirology. 2017;22 (6):1133-1139. doi:10.1111/resp.13035

22. Wewel AR, Gellermann I, Schwertfeger I, Morfeld M, Magnussen H, Jörres RA. Intervention by phone calls raises domiciliary activity and exercise capacity in patients with severe COPD. Respir Med. 2008;102(1):20-26. doi:10.1016/j.rmed.2007.08.011

23. Moy ML, Martinez CH, Kadri R, et al. Long-term effects of an internet-mediated pedometer-based walking program for chronic obstructive pulmonary disease: randomized controlled trial. $J$ Med Internet Res. 2016;18(8):e215. doi:10.2196/jmir.5622

24. Wan ES, Kantorowski A, Polak M, et al. Long-term effects of web-based pedometer-mediated intervention on COPD exacerbations. Respir Med. 2020;162:105878. doi:10.1016/j.rmed.2020.105878 
25. Osadnik C, Loeckx M, Louvaris Z, et al. The likelihood of improving physical activity after pulmonary rehabilitation is increased in patients with COPD who have better exercise tolerance. Int J Chron Obstruct Pulmon Dis. 2018;13:3515. doi:10.2147/COPD.S174827

26. McCarthy B, Casey D, Devane D, Murphy K, Murphy E, Lacasse Y. Pulmonary rehabilitation for chronic obstructive pulmonary disease. Cochrane Database Syst Rev. 2015;2:CD003793.
27. Gore S, Blackwood J, Guyette M, Alsalaheen B. Validity and reliability of accelerometers in patients with COPD: a systematic review. J Cardiopulm Rehabil Prev. 2018;38(3):147-158. doi:10.1097/ HCR.0000000000000284

\section{Publish your work in this journal}

The International Journal of COPD is an international, peer-reviewed journal of therapeutics and pharmacology focusing on concise rapid reporting of clinical studies and reviews in COPD. Special focus is given to the pathophysiological processes underlying the disease, intervention programs, patient focused education, and self management protocols. This journal is indexed on PubMed Central, MedLine and CAS. The manuscript management system is completely online and includes a very quick and fair peer-review system, which is all easy to use. Visit http://www.dovepress.com/testimonials.php to read real quotes from published authors. 